\title{
Failure of levofloxacin treatment in community-acquired pneumococcal pneumonia
}

\author{
Andrea Endimiani ${ }^{1}$, Gioconda Brigante ${ }^{1}$, Alessia A Bettaccini ${ }^{1}$, \\ Francesco Luzzaro1, Paolo Grossi² ${ }^{2}$ and Antonio Q Toniolo*1
}

\author{
Address: ${ }^{1}$ Laboratory of Microbiology and Virology, University of Insubria and Ospedale di Circolo e Fondazione Macchi, Varese, Italy and \\ ${ }^{2}$ Department of Infectious Diseases, University of Insubria and Ospedale di Circolo e Fondazione Macchi, Varese, Italy \\ Email: Andrea Endimiani - aendimiani@tin.it; Gioconda Brigante - giokybri@yahoo.it; Alessia A Bettaccini - mabodio@tin.it; \\ Francesco Luzzaro - francesco.luzzaro@ospedale.varese.it; Paolo Grossi - paolo.grossi@uninsubria.it; \\ Antonio Q Toniolo* - antonio.toniolo@ospedale.varese.it \\ * Corresponding author
}

Published: 24 November 2005

BMC Infectious Diseases 2005, 5:106 doi:10.1 186/147|-2334-5-106
Received: 29 July 2005

Accepted: 24 November 2005

This article is available from: http://www.biomedcentral.com/I47I-2334/5/I06

(c) 2005 Endimiani et al; licensee BioMed Central Ltd.

This is an Open Access article distributed under the terms of the Creative Commons Attribution License (http://creativecommons.org/licenses/by/2.0), which permits unrestricted use, distribution, and reproduction in any medium, provided the original work is properly cited.

\begin{abstract}
Background: Streptococcus pneumoniae is the leading cause of community-acquired pneumonia (CAP). High global incidence of macrolide and penicillin resistance has been reported, whereas fluoroquinolone resistance is uncommon. Current guidelines for suspected CAP in patients with co-morbidity factors and recent antibiotic therapy recommend initial empiric therapy using one fluoroquinolone or one macrolide associated to other drugs (amoxicillin, amoxicillin/clavulanate, broad-spectrum cephalosporins). Resistance to fluoroquinolones is determined by efflux mechanisms and/or mutations in the parC and parE genes coding for topoisomerase IV and/or gyrA and gyrB genes coding for DNA gyrase. No clinical cases due to fluoroquinolone-resistant $S$. pneumoniae strains have been yet reported from Italy.
\end{abstract}

Case presentation: A 72-year-old patient with long history of chronic obstructive pulmonary disease and multiple fluoroquinolone treatments for recurrent lower respiratory tract infections developed fever, increased sputum production, and dyspnea. He was treated with oral levofloxacin (500 mg bid). Three days later, because of acute respiratory insufficiency, the patient was hospitalized. Levofloxacin treatment was supplemented with piperacillin/tazobactam. Microbiological tests detected a S. pneumoniae strain intermediate to penicillin (MIC, I mg/L) and resistant to macrolides (MIC $>256 \mathrm{mg} / \mathrm{L}$ ) and fluoroquinolones (MIC $>32 \mathrm{mg} / \mathrm{L}$ ). Point mutations were detected in gyrA (Ser8I-Phe), parE (lle460-Val), and parC gene (Ser79-Phe; LysI37-Asn). Complete clinical response followed treatment with piperacillin/tazobactam.

Conclusion: This is the first Italian case of community-acquired pneumonia due to a fluoroquinolone-resistant $S$. pneumoniae isolate where treatment failure of levofloxacin was documented. Molecular analysis showed a group of mutations that have not yet been reported from Italy and has been detected only twice in Europe. Treatment with piperacillin/tazobactam appears an effective means to inhibit fluoroquinolone-resistant strains of $S$. pneumoniae causing community-acquired pneumonia in seriously ill patients. 


\section{Background}

Streptococcus pneumoniae is the leading cause of community-acquired pneumonia (CAP) and a major cause of meningitis and otitis media. Recent reports show an high global incidence of macrolide and penicillin resistance, whereas fluoroquinolone (FQ) resistance is not frequent [1-3]. Current guidelines for suspected bacterial CAP in patients with co-morbidity factors and recent antibiotic therapy recommend initial empiric therapy using one respiratory FQ or one macrolide associated to other drugs (amoxicillin, amoxicillin/clavulanate, broad-spectrum cephalosporins) $[4,5]$.

Resistance to FQ in S. pneumoniae is determined by efflux mechanisms and/or mutations in the quinolone resistance-determining regions (QRDRs) of parC and parE genes coding for topoisomerase IV and/or gyrA and gyrB genes coding for DNA gyrase [6]. The most frequent resistance mechanism detected in S. pneumoniae is represented by associated mutations of both gyrA and parC genes $[1,3,7]$. A single parC mutation appears to represent the first step conferring low-level FQ resistance [6]. A second mutational step in the gyrA gene produces high-level resistance to FQ [6]. Both mutations are probably selected in response to the widespread use of FQ $[8,9]$. Ciprofloxacin and levofloxacin use is commonly associated with parC gene mutations, whereas use of other FQ appears to favor the selection of mutations in other genes [6]. Here, we report the first Italian case of CAP in which failure of levofloxacin treatment was associated to highlevel FQ resistance in $S$. pneumoniae.

\section{Case presentation Microbiological methods}

Bacterial identification (ID) and antimicrobial susceptibility testing (AST) were achieved with the Phoenix System using SMIC/ID-2 panels (Becton Dickinson Diagnostic Systems, Sparks, MD). Specie ID was confirmed using optochin and bile solubility tests (Taxo Disc, BBL, Becton Dickinson) [10]. Determinations of minimal inhibitory concentration (MIC) were obtained with the Etest method (AB Biodisk, Solna, Sweden) on MuellerHinton agar with 5\% sheep blood (Oxoid, Milano, Italy). MIC values were interpreted according to current criteria of the Clinical and Laboratory Standards Institute [11].

Amplification of gyrA, gyrB,parC, parE, and pneumolysin genes were performed by PCR using reported oligonucleotides [12-14]. Direct DNA sequencing was obtained using the ABI Prism Big Dye terminator Cycle Sequencing Kit and the ABI Prism 310 sequencer (Applied Biosystems, Foster City, CA). DNA sequences were compared with sequences present in the GenBank database (gyrA, accession No. AB010387; gyrB, accession No. Z67740; parE and parC, accession No. Z67739). Efflux mechanisms were investigated by broth dilution in Mueller-Hinton medium containing 5\% sheep blood using serial dilution of ciprofloxacin in the presence or in the absence of $10 \mathrm{mg} / \mathrm{L}$ reserpine (Sigma-Aldrich, St. Louis, MO). A fourfold or greater decrease of the ciprofloxacin MIC in the presence of reserpine was considered as indicator of efflux mechanisms [15].

\section{Clinical data and results}

In March 2005, a 72-year-old patient with long history of chronic obstructive pulmonary disease (COPD), congenital bullous emphysema, and aortic valve incompetence developed abrupt-onset fever, cough, increased sputum production, and dyspnea. Over the past few years, solely on the basis of clinical data, the patient had received multiple treatments with oral levofloxacin and ciprofloxacin for recurrent lower respiratory tract infections (LRTi). At the onset, the family physician prescribed oral levofloxacin (500 mg, bid) and paracetamol (500 mg, tid). Three days later, because of non-remitting fever and acute respiratory insufficiency, the patient was admitted to the Emergency Room of the Ospedale di Circolo e Fondazione Macchi (Varese, Italy). On the basis of physical examination and hypoxemia $\left(\mathrm{pO}_{2}=45.4 \mathrm{~mm} \mathrm{Hg}\right)$ the patient was intubated. A central venous catheter was implanted. Blood analysis showed the following results: erythrocytes $4.23 \times 10^{6} / \mu \mathrm{L}$, hemoglobin $13.3 \mathrm{~g} / \mathrm{dL}$, hematocrit $38.8 \%$, platelets $201 \times 10^{3} / \mu \mathrm{L}$, total leukocytes $15.75 \times 10^{3} / \mu \mathrm{L}$ (neutrophils 78.2\%, lymphocytes $12.1 \%$ ), ESR (erythrocyte sedimentation rate) $86.2 \mathrm{~mm} / \mathrm{h}$, CRP (C-reactive protein) $118 \mathrm{mg} / \mathrm{L}$, creatinine $1.02 \mathrm{mg} / \mathrm{dL}$, BUN (blood urea nitrogen) $26 \mathrm{mg} / \mathrm{dL}$. Chest radiograph showed accentuated peribronchial interstitium and hilar expansion. Intravenous corticosteroids and bronchodilators were given. Twelve hours later, the patient was transferred to the Intensive Care Unit (ICU) where 4 bronchoalveolar lavages (BAL) were obtained. Levofloxacin was continued by the i.v. route (500 $\mathrm{mg}$, bid) supplemented with piperacillin/tazobactam (2.25 g tid). Serial BAL were also obtained during the first 3 days of hospitalization.

Microbiological tests detected a multidrug-resistant (MDR) S. pneumoniae isolate $\left(10^{6}\right.$ colony forming units/ $\mathrm{mL}$ ) in the four BAL samples obtained at admission. Isolates had the following MIC values: penicillin G $(1 \mathrm{mg} / \mathrm{L})$, piperacillin $(1 \mathrm{mg} / \mathrm{L})$, piperacillin/tazobactam $(1 \mathrm{mg} / \mathrm{L})$, ceftriaxone $(0.5 \mathrm{mg} / \mathrm{L})$, cefotaxime $(0.5 \mathrm{mg} / \mathrm{L})$, cefepime $(0.5 \mathrm{mg} / \mathrm{L})$, imipenem $(0.064 \mathrm{mg} / \mathrm{L})$, erythromycin $(>256$ $\mathrm{mg} / \mathrm{L})$, claritromicyn $(>256 \mathrm{mg} / \mathrm{L})$, clindamycin $(>256$ $\mathrm{mg} / \mathrm{L} \mathrm{mg} / \mathrm{L})$, levofloxacin $(>32 \mathrm{mg} / \mathrm{L})$, ciprofloxacin $(>32$ $\mathrm{mg} / \mathrm{L})$, moxifloxacin $(>32 \mathrm{mg} / \mathrm{L})$, tetracycline $(0.5 \mathrm{mg} / \mathrm{L})$, chloramphenicol $(16 \mathrm{mg} / \mathrm{L})$, trimethoprim-sulfamethoxazole $(4 \mathrm{mg} / \mathrm{L})$, rifampin $(0.5 \mathrm{mg} / \mathrm{L})$ vancomycin $(0.25$ $\mathrm{mg} / \mathrm{L})$, teicoplanin $(0.125 \mathrm{mg} / \mathrm{L})$, linezolid $(0.75 \mathrm{mg} / \mathrm{L})$, and telithromycin $(\leq 0.006 \mathrm{mg} / \mathrm{L})$. 
By direct DNA sequencing, point mutations in the gyrA (Ser81-Phe) and parE (Ile460-Val) genes were detected in all isolates. Two point mutations of the parC gene (Ser79Phe; Lys137-Asn) were also present. No mutations were detected in the gyrB gene. The reserpine response assay failed to evidence FQ efflux. The pneumolysin gene was detected in all isolates.

Prompt clinical response followed the empirical treatment given at the ICU. On day-two, the patient was afebrile, hypoxemia resolved $\left(\mathrm{pO}_{2}=157 \mathrm{~mm} \mathrm{Hg}\right)$, inflammatory markers were decreased (total leukocytes $7.19 \times 10^{3} / \mu \mathrm{L}$, neutrophils $76.1 \%$, lymphocytes $12.0 \%$, ESR $45 \mathrm{~mm} / \mathrm{h}$, CRP $57 \mathrm{mg} / \mathrm{L}$ ), and blood cultures were negative. BAL samples obtained on day- 2 and at subsequent times were negative. Five days after hospital admission, levofloxacin was suspended and piperacillin/ tazobactam was reduced ( $1.25 \mathrm{~g}$ tid). Respiratory assistance was stopped on day-12. Antibiotic treatment ended on day-14. Complete recovery ensued and the patient was discharged on day-16.

\section{Conclusion}

The impact of antibiotic resistance on the treatment outcome of patients with CAP is a matter of discussion [4]. FQ are often the primary choice for empirical treatment of patients with co-morbidity factors such as COPD, diabetes, and renal or congestive heart failure [4,5]. A few reports show an association between FQ resistance and treatment failure in CAP caused by $S$. pneumoniae $[16,17]$. No cases have been yet reported from Italy, possibly due to the low incidence of FQ-resistant strains [3].

The present report describes the first Italian case of CAP due to a FQ-resistant strain of $S$. pneumoniae. Failure of oral levofloxacin was observed. According to current criteria [11], the isolate was MDR i.e., intermediate to penicillin, resistant to chloramphenicol, trimethoprimsulfamethoxazole, macrolides, clindamycin and FQ. The strain showed single mutations of gyrA and parE genes associated with two point-mutations of the parC gene. High-level resistance to FQ resulted (MIC >32 mg/L). This justified the initial treatment failure when the patient was given levofloxacin alone. Molecular analysis showed a group of mutations that have not yet been reported from Italy $[3,18]$. Association of the reported mutations in gyrA, parE and parC genes has been detected only twice in Europe [3], but is relatively more frequent in the USA [7]. As demonstrated by studies of $S$. pneumoniae strains exposed in vitro to FQ, ciprofloxacin and levofloxacin use is commonly associated with parC gene mutations, whereas use of other FQ appears to favor the selection of mutations in other genes [6].
The reported clinical case suggests that the following precautions need to be used for patients with specific LRTi risk factors: i) before therapy, respiratory samples should be obtained to identify the responsible agents and to define antimicrobial susceptibility [16]; ii) patients previously treated with FQ may be given these drugs, but need strict clinical monitoring during the first 3 days of therapy to evaluate the clinical response [19]; iii) since only $1 \%$ of FQ-resistant S. pneumoniae strains are also resistant to broad-spectrum beta-lactams, seriously ill patients can be safely treated with combinations of FQ plus broad-spectrum beta-lactams [20]. Empiric treatment of CAP in patients seriously compromised using piperacillin/tazobactam (as in the reported case) appears an effective means to inhibit FQ-resistant strains of S. pneumoniae. In particular, piperacillin/tazobactam appears indicated for ICU patients since, in contrast to monotherapy with extended-spectrum cephalosporins (e.g., ceftriaxone, cefotaxime), does not favor the selection of ESBL-positive enterobacteria and/or chromosomal beta-lactamase hyperproducers (e.g., Pseudomonas aeruginosa) [21,22]. Additionally, this drug's spectrum is wider than that of $3^{\text {rd }}-4^{\text {th }}$ generation cephalosporins.

In Italy FQ consumption is higher than in the rest of Europe [8]. Thus, it seems possible that over the next years an increased prevalence of FQ-resistant $S$. pneumoniae strains will be observed. For this reason the detection of a community-acquired MDR isolate of S. pneumoniae is of special concern. Emerging resistance traits in this species underline the need of in vitro tests based on MIC data in order to select the most appropriate drugs for preventing the dissemination of epidemic clones.

\section{Competing interests}

The author(s) declare that they have no competing interests.

\section{Authors' contributions}

$\mathrm{AE}$ and GB performed microbiological tests, analyzed the data and prepared the manuscript.

AAB carried out molecular assays.

PG diagnosed, cured and provided clinical data of the patient.

FL and AQT coordinated the study and helped writing the manuscript.

All authors read and approved the final version of the manuscript.

\section{Acknowledgements}

We wish to thank Vito Elia, and Silvano Colella for their expert technical assistance. Written consent was obtained from the patient for publication 
of the study. This work was supported by a PRIN grant from the Italian Ministry for Education, University, and Research (MIUR, Rome, Italy). AE is a Ph.D. student of the University of Insubria, Varese, Italy.

\section{References}

I. Canton R, Morosini M, Enright MC, Morrissey I: Worldwide incidence, molecular epidemiology and mutations implicated in fluoroquinolone-resistant Streptococcus pneumoniae : data from the global PROTEKT surveillance programme. J Antimicrobial Chemother 2003 , 52:944-952.

2. Brown SD, Farrell DJ, Morrissey I: Prevalence and molecular analysis of macrolide and fluoroquinolone resistance among isolates of Streptococcus pneumoniae collected during the 2000-200I PROTEKT US Study. J Clin Microbiol 2004, 42:4980-4987.

3. Reinert RR, Reinert S, van der Linden M, Cil MY, Al-Lahham A, Appelbaum P: Antimicrobial susceptibility of Streptococcus pneumoniae in eight European countries from 2001 to 2003. Antimicrob Agents Chemother 2005, 49:2903-29I3.

4. Mandell LA, Bartlett JG, Dowell SF, File TM Jr, Musher DM, Whitney $C$ : Update of practice guidelines for the management of community-acquired pneumonia in immunocompetent adults. Clin Infect Dis 2003, 37:|405-|433.

5. Gilbert DN, Moellering RC Jr, Eliopoulos GM, Sande MA: The Sanford guide to antimicrobial therapy Thirty-fifth edition. Hyde Park, VT, USA; 2005.

6. Eliopoulos GM: Quinolone resistance mechanisms in pneumococci. Clin Infect Dis 2004:350-356.

7. Pletz MWR, McGee L, Jorgensen J, Beall B, Facklam RR, Whitney CG Klugman KP, the Active Bacterial Core Surveillance Team: Levofloxacin-resistant invasive Streptococcus pneumoniae in the United States: evidence for clonal spread and the impact of conjugate pneumococcal vaccine. Antimicrob Agents Chemother 2004, 48:349I-3497.

8. Goossens H, Ferech M, Vander Stichele R, Elseviers M, for the ESAC Project Group: Outpatient antibiotic use in Europe and association with resistance: a cross-national database study. Lancet 2005, 365:579-587.

9. Pérez-Trallero E, Marimón JM, González A, Ercibengoa M, Larruskain $\mathrm{J}$ : In vivo development of high-level fluoroquinolone resistance in Streptococcus pneumoniae in chronic obstructive pulmonary disease. Clin Infect Dis 2005, 41:560-564.

10. Arbique JC, Poyart C, Trieu-Cuot P, Quesne G, da Glória S, Carvalho M, Steigerwalt AG, Morey RE, Jackson D, Davidson RJ, Facklam RR: Accuracy of phenotypic and genotypic testing for identification of Streptococcus pneumoniae and description of Streptococcus pseudopneumoniae sp. nov. J Clin Microbiol 2004, 42:4686-4696.

II. Clinical and Laboratory Standards Institute: Performance standards for antimicrobial susceptibility testing 2005.

12. Janoir C, Zeller V, Kitzis M-D, Moreau NJ, Gutmann L: High-leve fluoroquinolone resistance in Streptococcus pneumoniae requires mutations in parC and gyrA. Antimicrob Agents Chemother 1996, 40:2760-2764.

13. Perichon B, Tankovic J, Courvalin P: Characterization of a mutation in the parE gene that confers fluoroquinolone resistance in Streptococcus pneumoniae. Antimicrob Agents Chemother 1997, 4I:1166-1167

14. Dagan R, Shriker O, Hazan I, Leibovitz E, Greenberg D, Schlaeffer F, Levy R: Prospective study to determined clinical relevance of detection of pneumococcal DNA in sera of children by PCR. J Clin Microbiol 1998, 36:669-673.

15. Bast DJ, Low DE, Duncan CL, Kilburn L, Mandell LA, Davidson RJ, de Azavedo JCS: Fluoroquinolone resistance in clinical isolates of Streptococcus pneumoniae : contributions of type II topoisomerase mutations and efflux to levels of resistance. Antimicrob Agents Chemother 2000, 44:3049-3054.

16. Davidson R, Cavalcanti R, Brunton JL, Bast DJ, de Azavedo JCS, Kibsey $P$, Fleming C, MLT , Low DE: Resistance to levofloxacin and failure of treatment of pneumococcal pneumonia. $N$ Engl J Med 2002, 346:747-750.

17. Fuller JD, Low DE: A review of Streptococcus pneumoniae infection treatment failures associated with fluoroquinolone resistance. Clin Infect Dis 2005, 4 I:I I8-I 2 I.
18. Montanari MP, Tili E, Cochetti I, Mingoia M, Manzin A, Varaldo PE: Molecular characterization of clinical Streptococcus pneumoniae isolates with reduced susceptibility to fluoroquinolones emerging in Italy. Microb Drug Resist 2004, I 0:209-2 I 7.

19. Anderson KB, Tan JS, File TM Jr, DiPersio JR, Willey BM, Low DE: Emergence of levofloxacin-resistant pneumococci in immunocompromised adults after therapy for communityacquired pneumonia. Clin Infect Dis 2003, 37:376-38I.

20. Jones RN, Fritsche TR, Sader HS: Therapeutic options among broad-spectrum $\beta$-lactam for infections caused by levofloxacin-nonsusceptible Streptococcus pneumoniae. Diagn Microbiol Infect Dis 2005, 52:129-133.

21. Kollef $\mathrm{MH}$ : Optimizing antibiotic therapy in the intensive care unit setting. Critical Care 2001, 5:189-195.

22. Graffunder EM, Preston KE, Evans AM, Venezia RA: Risk factors associated with extended-spectrum $\beta$-lactamase-producing organisms at a tertiary care hospital. I Antimicrob Chemother 2005, 56:139-145.

\section{Pre-publication history}

The pre-publication history for this paper can be accessed here:

http://www.biomedcentral.com/1471-2334/5/106/pre pub
Publish with Biomed Central and every scientist can read your work free of charge

"BioMed Central will be the most significant development for disseminating the results of biomedical research in our lifetime. "

Sir Paul Nurse, Cancer Research UK

Your research papers will be:

- available free of charge to the entire biomedical community

- peer reviewed and published immediately upon acceptance

- cited in PubMed and archived on PubMed Central

- yours - you keep the copyright 